\title{
The volume and time comparison principle and transition probability estimates for random walks
}

\author{
András Telcs \\ Budapest University of Technology and Economics, Department of Computer Science and Information Theory, Mûegyetem \\ rkp. 3-9, H-1111, Budapest, Hungary \\ h197teleella.hu
}

This paper presents necessary and sufficient conditions for on- and off-diagonal transition probability estimates for random walks on weighted graphs. On the integer lattice and on may fractal type graphs both the volume of a ball and the mean exit time from a ball are independent of the center, uniform in space. Here the upper estimate is given without such restriction and two-sided estimate is given if the mean exit time is independent of the center but the volume is not.

Keywords: random walks, heat kernel estimates

\section{Introduction}

This paper presents on- and off-diagonal transition probability estimates on weighted graphs. The central object of the investigation is the minimal solution of the discrete heat equation

$$
\triangle u_{n}=\frac{\partial}{\partial n} u_{n}
$$

on weighted graphs, where $\triangle=P-I$ is the discrete Laplace and $\frac{\partial}{\partial n} u_{n}=u_{n+1}-u_{n}$ is the differential operator. The classical form of the diagonal upper estimate for the minimal solution is

$$
p_{n}(x, x) \leq C n^{-\frac{d}{2}},
$$

which holds on $\mathbb{Z}^{d}, d \in \mathbb{N}$, where $V(x, R)$ the volume of a ball of radius $R$ has uniformly polynomial growth $V(x, R) \simeq R^{d}$. Coulhon and Grigor'yan [4] proved diagonal estimates for graphs for non-uniform volume. They have shown that the following tree conditions are equivalent. 1.

$$
p_{n}(x, x) \leq \frac{C}{V(x, \sqrt{n})}
$$

1365-8050 @ 2003 Discrete Mathematics and Theoretical Computer Science (DMTCS), Nancy, France 
in conjunction with volume doubling property.

2 .

$$
p_{n}(x, y) \leq \frac{C}{V(x, \sqrt{n})} \exp \left(-c \frac{d^{2}(x, y)}{n}\right)
$$

plus the volume doubling property.

3.The relative Faber-Krahn inequality.

For a detailed introduction and history see Woess [22] (or Barlow [1], Coulhon [3], Grigor'yan [10], Varopoulos, Saloff-Coste, Coulhon [21]).

Another branch of the generalization was the study of fractals and fractal like graphs cf. [1] to obtain sub-Gaussian estimates

$$
p_{n}(x, y) \simeq \frac{C}{n^{\frac{\alpha}{\beta}}} \exp \left(-\frac{d^{\beta}(x, y)}{C n}\right)^{\frac{1}{\beta-1}}
$$

where the constant $C$ different for the upper and lower bound. Here the sub-Gaussian feature is provided by the $\beta>2$ exponent which describes the mean exit time

$$
E(x, R) \simeq R^{\beta}
$$

of a ball $B(x, R)$. Let us consider the (generalized) inverse function $e(x, n)$ of the mean exit time $E(x, R)$ in the second variable. Based on the usual heuristic one might expect that a fully local diagonal upper estimate of the form of

$$
p_{n}(x, x) \leq \frac{C}{V(x, e(x, n))}
$$

can be given. This paper announces on- and off- diagonal estimates of this local type. The sub-Gaussian exponents of the off-diagonal upper and lower estimates do not coincide in this generality ( for further explanation and examples see [14]). It can be seen that the sub-Gaussian exponents meet if and only if the mean exit time is uniform in the space i.e.

$$
E(x, R) \simeq F(R)
$$

for a function $F$. This is usually called in the physics literature space-time scale function and a semi-local framework can be developed in its presence.

\section{Preliminaries}

Let us consider a countable infinite connected graph $\Gamma$. A weight function $\mu_{x, y}=\mu_{y, x}>0$ is given on the edges $x \sim y$. This weight induces a measure $\mu(x)$

$$
\begin{aligned}
\mu(x) & =\sum_{y \sim x} \mu_{x, y} \\
\mu(A) & =\sum_{y \in A} \mu(y)
\end{aligned}
$$

on the vertex set $A \subset \Gamma$ and defines a reversible Markov chain $X_{n} \in \Gamma$, i.e. a random walk on the weighted graph $(\Gamma, \mu)$ with transition probabilities

$$
P(x, y)=\frac{\mu_{x, y}}{\mu_{(x)}}
$$




$$
P_{n}(x, y)=\mathbb{P}\left(X_{n}=y \mid X_{0}=x\right) .
$$

Condition 1 In the whole sequel we assume that condition $\left(\mathbf{p}_{0}\right)$ holds, that is, there is a universal $p_{0}>0$ such that for all $x, y \in \Gamma, x \sim y$

$$
\frac{\mu_{x, y}}{\mu(x)} \geq p_{0} .
$$

The graph is equipped with the usual (shortest path length) graph distance $d(x, y)$ and open metric balls are defined for $x \in \Gamma, R>0$ as $B(x, R)=\{y \in \Gamma: d(x, y)<R\}$ and its $\mu$-measure is denoted by $V(x, R)$.

Definition 2.1 The weighted graphs satisfies the volume comparison principle (VC) (c.f. [13]) if there is a constant $C_{V}>1$ such that for all $x \in \Gamma$ and $R>0, y \in B(x, R)$

$$
\frac{V(x, 2 R)}{V(y, R)} \leq C_{V}
$$

Definition 2.2 The weighted graph has the volume doubling (VD) property if there is a constant $D_{V}>0$ such that for all $x \in \Gamma$ and $R>0$

$$
V(x, 2 R) \leq D_{V} V(x, R) .
$$

One can see that $(V D)$ and $(V C)$ are equivalent.

\section{Upper estimates}

This section is mainly devoted to upper estimates, but at the end lower estimates are also given providing comparison with the upper one..

Let us consider the exit time $T_{B(x, R)}=\min \left\{k>0: X_{k} \notin B(x, R)\right\}$ from the ball $B(x, R)$ and its mean value $E_{z}(x, R)=\mathbb{E}\left(T_{B(x, R)} \mid X_{0}=z\right)$ and let us use the $E(x, R)=E_{x}(x, R)$ short notation. In the analogy to the volume comparison we introduce the (mean exit) time comparison principle.

Definition 3.1 We will say that the weighted graph $(\Gamma, \mu)$ satisfies the time comparison principle (TC) if there is a constant $C_{T}>1$ such that for all $x \in \Gamma$ and $R>0, y \in B(x, R)$

$$
\frac{E(x, 2 R)}{E(y, R)} \leq C_{T}
$$

Definition 3.2 We will say that $(\Gamma, \mu)$ has the time doubling property (TD) if there is a $D_{T}>0$ such that for all $x \in \Gamma$ and $R \geq 0$

$$
E(x, 2 R) \leq D_{T} E(x, R) .
$$

One should notice that $(T C)$ implies $(T D)$ but the opposite is not true in general. Basically the $(V C)$ and $(T C)$ principles specify the framework of the local setup for our study. We introduce the skewed version of the parabolic mean value inequality.

Definition 3.3 We shall say that the skewed parabolic mean value inequality ( $s P M V$ ) holds if there are $0<c_{1}<c_{2} \leq 1 \leq C$ such that for all $R>0, x \in \Gamma, y \in B(x, R)$ for all non-negative solutions $u_{n}$ of the discrete heat equation on $\left[0, c_{2} E(x, R)\right] \times B(x, R)$

$$
u_{n}(x) \leq \frac{C}{V(y, 2 R) E(y, 2 R)} \sum_{i=c_{1} E}^{n} \sum_{z \in B(x, R)} u_{i}(z) \mu(z)
$$


satisfied, where $E=E(x, R), n=c_{2} E$.

Definition 3.4 We shall say that the mean value inequality (MV) holds, i.e. for all $x \in \Gamma, R>0$ and for all function $u \geq 0$ on $\bar{B}(x, R)$ which is harmonic on $B=B(x, R)$

$$
u(x) \leq \frac{C}{V(x, R)} \sum_{y \in B} u(y) \mu(y)
$$

Definition 3.5 The local kernel function $1 \leq k=k_{y}=k_{y}(n, R) \leq n$, is defined as the maximal integer for which

$$
\frac{n}{k} \leq q E\left(y,\left\lfloor\frac{R}{k}\right\rfloor\right)
$$

or $k=0$ by definition if there is no appropriate $k$. Here $q$ is a small fixed constant.

Definition 3.6 For convenience we will use the following notation

$$
k_{C}(x, n, R)=\min _{z \in B(x, e(x, n))}\left\{k_{z}\left(C n, \frac{1}{C} R\right)\right\} .
$$

Denote $R=d(x, y)$ and let

$$
\kappa_{C}(n, x, y)=\max \left\{k_{C}(x, n, R), k_{C}(y, n, R)\right\}
$$

if $r>3[e(x, n)+e(y, n)]$ and $\kappa_{C}=0$ otherwise.

Theorem 3.1 If $(\Gamma, \mu)$ satisfies $\left(p_{0}\right)$ then the following conditions are equivalent.

1. (sPMV) holds,

2. $(V C),(T C)$ and $(M V)$,

3. $(V C),(T C)$ and the local diagonal upper estimate holds:

$$
P_{n}(x, x) \leq \frac{C \mu(x)}{V(x, e(x, n))}
$$

4. $(V C),(T C)$ and the local upper estimate holds:

$$
p_{n}(x, y) \leq \frac{C}{\sqrt{V(x, e(x, n)) V(y, e(y, n))}} \exp \left(-c \kappa_{3}(n, x, y)\right)
$$


The proof of the diagonal upper estimate can be given along the lines of [12] while the off-diagonal estimate based on a generalization of an inequality due to Davies [6].

Remark 3.1 It can also be shown that $\left(p_{0}\right)$ and $(T C)$ imply

$$
p_{2 n}(x, x) \geq \frac{c}{V(x, e(x, 2 n))} .
$$

One gets a weaker upper estimate introducing

$$
\kappa(n, x, y)=\min _{z \in A_{x, y}}\left\{k_{z}\left(3 n, \frac{1}{3} d(x, y)\right)\right\}
$$

where $A_{x, y}=B(x, d(x, y)) \cup B(y, d(x, y))$ if $d(x, y)>3[e(x, n)+e(y, n)]$ and $\kappa(n, x, y)=0$ otherwise. Similarly we introduce

$$
l(n, x, y)=\max _{z \in A_{x, y}}\left\{k_{z}(n, d(x, y))\right\} .
$$

Let us measure the inhomogeneity of the mean exit time for any $A \subset \Gamma$ by

$$
\delta(n, A)=\log \left[\max _{z, v \in A} \frac{e(z, n)}{e(v, n)}\right]
$$

and denote the lower sub-Gaussian kernel by $\mathrm{v}$;

$$
\mathrm{v}(n, x, y)=l(n, x, y)\left[1+\delta\left(n, A_{x, y}\right)\right] .
$$

Definition 3.7 The weighted graph $(\Gamma, \mu)$ satisfies the elliptic Harnack inequality $(\mathbf{H})$ if there is a $C>0$ such that for all $x \in \Gamma$ and $R>0$ and for all $u \geq 0$ on $\bar{B}(x, 2 R)$ harmonic functions on $B(x, 2 R)$ which means that

$$
P u=u
$$

on $B(x, 2 R)$, the following inequality holds

$$
\max _{B(x, R)} u \leq C \min _{B(x, R)} u
$$

Using the above notations the following statement can be given, which on the upper estimate side is direct consequence of the above results observing that the elliptic Harnack inequality implies the mean value inequality $(M V)$.

Theorem 3.2 Assume that $(\Gamma, \mu)$ satisfies $\left(p_{0}\right),(V C),(T C)$ and the elliptic Harnack inequality $(H)$, then

$$
p_{n}(x, y) \leq \frac{C}{\sqrt{V(x, e(x, n)) V(y, e(y, n))}} \exp (-c \kappa(n, x, y)),
$$

and

$$
\widetilde{p}_{n}(x, y) \geq \frac{c}{V(x, e(x, n))} \exp (-C v(n, x, y))
$$

where $\widetilde{p}_{n}=p_{n+1}+p_{n}$.

Remark 3.2 One can rewrite (3.33) in the form of

$$
\widetilde{p}_{n}(x, y) \geq \frac{c}{\sqrt{V(x, e(x, n)) V(y, e(y, n))}} \exp (-C v(n, x, y)),
$$

to be compared to (3.32). 
The lower estimate is proved via an important intermediate estimate (called near diagonal lower estimate c.f. [12] or [19]) then the standard Aronson's chaining argument can be used.

Remark 3.3 One should recognize that the upper and lower estimate rely on comparison of volume and exit times of a chain of balls connecting $x$ and $y$. If the mean exit time is basically independent of the center of the ball it is clear from the definitions that $k \simeq l, \delta \simeq 1$ and hence $\kappa \simeq v$ which means that the upper and lower estimate are the same up to the constants.

\section{Two-sided estimates}

The semilocal framework is received from the local one if we assume that.

$$
E(x, R) \simeq E(y, R)
$$

for all $x, y \in \Gamma$. The study of semi-local situation starts with the investigation of the space-time scale function $F(R), R \geq 0$ which is

$$
F(R)=\inf _{x \in \Gamma} E(x, R) .
$$

¿From $(E)$ it follows that $F$ satisfies with a fixed $C_{0}>1$ for all $x \in \Gamma$ and $R \geq 0$

$$
F(R) \leq E(x, R) \leq C_{0} F(R) .
$$

Function $F$ inherits certain properties of $E(x, R)$, among others from $(T D)$ it follows that

$$
F(2 R) \leq D_{F} F(R) .
$$

The inherited properties are referred by the notation $\left(E D_{F}\right)$ (c.f. [19]). This function takes over the role of $R^{\beta}$ ( or $R^{2}$ ). The inverse function of $F, f()=.F^{-1}($.$) takes over the role of R^{\frac{1}{\beta}}\left(R^{\frac{1}{2}}\right)$ in the (sub-)Gaussian estimates.

Definition 4.1 The transition probability satisfies the sub-Gaussian upper estimate $\left(\mathbf{U E}_{F}\right)$ with respect to $F$ if there are $c, C>0$ such that

$$
P_{n}(x, y) \leq \frac{C \mu(y)}{V(x, f(n))} \exp -c k(n, d(x, y))
$$

and the sub-Gaussian lower estimate $\left(\mathbf{L E}_{F}\right)$ is satisfied if

$$
\widetilde{P}_{n}(x, y) \geq \frac{c \mu(y)}{V(x, f(n))} \exp -C k(n, d(x, y)),
$$

where $\widetilde{P}_{n}=P_{n}+P_{n+1}$ and the kernel function $k=k(n, R) \geq 1$, is defined as the maximal integer for which

$$
\frac{n}{k} \leq q F\left(\left\lfloor\frac{R}{k}\right\rfloor\right)
$$

or $k=0$ by definition if there is no appropriate $k$. 
As we indicated the parabolic and elliptic Harnack inequalities play important role in the study of two-sided bound of the heat kernel. Here we give the formal definitions of the parabolic one.

Definition 4.2 The weighted graph $(\Gamma, \mu)$ satisfies the ( $F$-parabolic or simply) parabolic Harnack inequality $\left(\mathbf{P H}_{F}\right)$ if the following condition holds. There is a $C_{H}>0$ constant such that for any solution $u \geq 0$ of the equation

$$
u_{n+1}(x)=P u_{n}(x)
$$

on $\mathcal{U}=[k, k+F(4 R)] \times B(x, 2 R)$ for $k, R \in \mathbb{N}$ the following is true. On the smaller cylinders defined by

$$
\begin{aligned}
\mathcal{U}^{-} & =[k+F(R), k+F(2 R)] \times B(x, R) \\
\text { and } \mathcal{U}^{+} & =[k+F(3 R), k+F(4 R)] \times B(x, R)
\end{aligned}
$$

and taking $\left(n_{-}, x_{-}\right) \in \mathcal{U}^{-},\left(n_{+}, x_{+}\right) \in \mathcal{U}^{+}, d\left(x_{-}, x_{+}\right) \leq n_{+}-n_{-}$the inequality

$$
u\left(n_{-}, x_{-}\right) \leq C_{H} \widetilde{u}\left(n_{+}, x_{+}\right)
$$

holds, where $\widetilde{u}_{n}=u_{n}+u_{n+1}$ short notation was used. The elliptic Harnack inequality is a direct consequence of the F-parabolic one as it is true for the classical case.

Based on the above definitions the following theorem can be formulated.

Theorem 4.1 If a weighted graph $(\Gamma, \mu)$ satisfies $\left(p_{0}\right)$ then the following statements are equivalent.

1. $\exists F$ satisfies $\left(E D_{F}\right)$ and the $F$-parabolic Harnack inequality $\left(P H_{F}\right)$,

2. $\exists F$ satisfies $\left(E D_{F}\right),\left(U E_{F}\right)$ and $\left(L E_{F}\right)$,

3. $(V D),(T D),(E)$ and $(H)$ hold.

Theorem 3.2 implies in the semi-local framework the corresponding off-diagonal estimate ( see also Remark 3.3). The other implication have been proved in [19]. The presented results generalize several works, among others Moser [17], [18], Davies [5], Coulhon, Grigor'yan [4], Grigor'yan [9], Li,Yau [16], Varopoulos [20]),[7], Fabes, Stroock [8], Hebisch, Saloff-Coste [15]. Let us mention that in [15] the equivalence of $\left(\mathrm{PH}_{F}\right)$ and the $F$-based two-sided sub-Gaussian estimate was already shown.

\section{References}

[1] M.T. Barlow, Diffusion on Fractals, in:'Lectures on Probability Theory and Statistics, Ecole d'été de Probabilités de Saint-flour XXV -1995”, Lecture Notes Math. 1690, Springer 1998, 1-121

[2] M.T. Barlow, T. Coulhon, A. Grigor'yan, Manifolds and graphs with slow heat kernel decay, Invent. Math., 144 (2001) 609-649

[3] T. Coulhon, Analysis on infinite graphs with regular volume growth, JE 2070, No 17/18, November 1997, Université de Cergy-Pontoise

[4] T. Coulhon, A. Grigor'yan, Random walks on graphs with regular volume growth, Geometry and Functional Analysis, 8, (1998) 656-701 
[5] E.B. Davies, Heat kernels and spectral theory, Cambridge University Press, Cambridge, 1989

[6] E.B.Davies, Heat kernel bounds, conservation of probability and the Feller property, J. d'Analyse Math. 58. (1992), 99-119

[7] T. Delmotte, Parabolic Harnack inequality and estimates of Markov chains on graphs.Revista Matemática Iberoamericana 1,(1999), 181-232

[8] E. Fabes, D. Stroock, A new proof of the Moser's parabolic Harnack inequality using the old ideas of Nash, Arch. Rat. Mech. Anal., 96, (1986) 327-338

[9] A. Grigor'yan, The heat equation on non-compact Riemannian manifolds, (in Russian) Matem. Sbornik 182:1, (1991), 55-87 Engl. transl., Math. USSR db. 72:1 (1992) 47-77

[10] A. Grigor'yan, Heat kernels on manifolds, graphs and fractals, Prog. in Math., 201 (2001) 393-405

[11] A. Grigor'yan, A. Telcs, Sub-Gaussian estimates of heat kernels on infinite graphs, Duke Math. J., $109,3,(2001), 452-510$

[12] A. Grigor'yan, A. Telcs, Harnack inequalities and sub-Gaussian estimates for random walks, Math. Ann. 324. (2002) 521-556

[13] M. Gromov, Groups of polynomial growth and expanding maps. Publ. Math. Inst. H. Poincaré Probab. Statist. 53 (1981), 57-73

[14] B. Hambly, T. Kumagai, Heat kernel estimates for symmetric random walks ona class of fractal graphs and stability under rough isometries, preprint

[15] W. Hebisch, L. Saloff-Coste, On the relation between elliptic and parabolic Harnack inequalities, Ann. Inst. Fourier 51 (2001) 5, 1437-1481

[16] P. Li, S.-T. Yau, On the parabolic kernel of the Schrödinger operator , Acta Math. 156, (1986) $153-201$

[17] J. Moser, On Harnack's Theorem for elliptic differential equations, Communications of Pure and Applied Mathematics, 16, (1964) 101-134

[18] J. Moser, On Harnack's theorem for parabolic differential equations, Communications of Pure and Applied Mathematics, 24, (1971) 727-740

[19] A. Telcs, Volume and time doubling of graphs and random walk, the strongly recurrent case, Communication on Pure and Applied Mathematics, LIV, (2001), 975-1018

[20] R. Th. Varopoulos, Hardy-Littlewood theory for semigroups, J. Functional Analysis 63, (1985) 215239

[21] R. Th. Varopoulos, L. Saloff-Coste, T. Coulhon, Analysis and geometry on Groups, Cambridge University Press, 1993

[22] W. Woess, Random walks on infinite graphs and groups, Cambridge University Press, Cambridge, 2000 